\title{
PAEDIATRIC PROBLEMS - I
}

\author{
Caroline M Doig
}

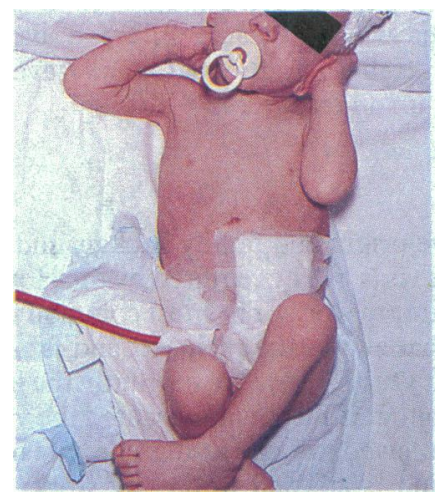

Infant with temporary

caecostomy.

\section{Constipation}

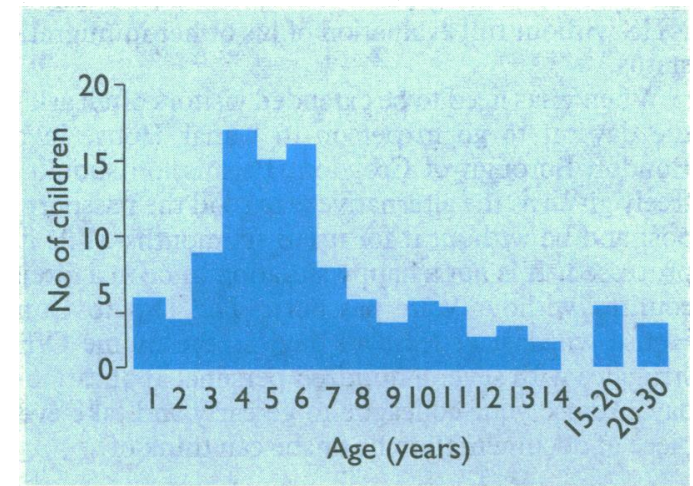

Age of children presenting with "idiopathic" constipation.
Constipation is the commonest cause of abdominal pain in childhood. A full rectum may compress the bladder, causing urinary problems. Constipation may be considered as either the passage of hard stools or an irregular bowel habit. Less easily recognisable is the passage of only small motions each day with the child retaining faeces. A palpable colon may be found on examination, but if the motion is soft radiography of the abdomen may be necessary to make the diagnosis.

If untreated three problems may arise: abdominal pain, anal fissure, and soiling because of overflow incontinence. The cause of constipation may be an inadequate diet, poor toilet facilities at home or more commonly at school, or previous illness or fever. Treatment involves dietary advice and toilet retraining. It may be necessary to empty the bowel by means of rectal washouts or enemas, or both, before giving laxatives, the dosage of which varies with each child. The general principles of treatment with laxatives include giving an adequate dosage for a long enough period of time, with gradual tailing off. Rarely a child may need admission to hospital for retraining.

Anal fissure is indicated by a history of pain on defecation with crying and "holding back" when on the toilet. Streaks of blood may be seen on toilet paper or the stool. A skin tag (sentinal pile) may indicate a fissure. Rectal examination should not be attempted if the diagnosis is obvious. Treatment entails oral laxatives for the constipation and applying local anaesthetic cream to the area. Suppositories should not be used. Occasionally anal stretch under general anaesthetic may be necessary to relieve sphincter spasm.
Abdominal radiograph showing severe constipation.
The anal tag is not the cause of the pain-it indicates the anal fissure, which is. 


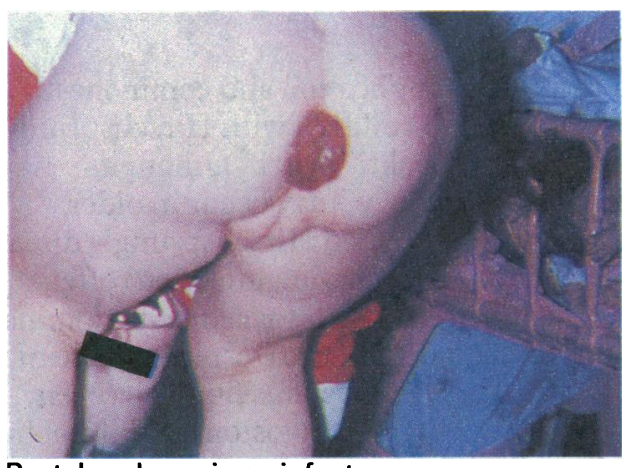

Rectal prolapse in an infant.

\section{Hirschsprung's disease}

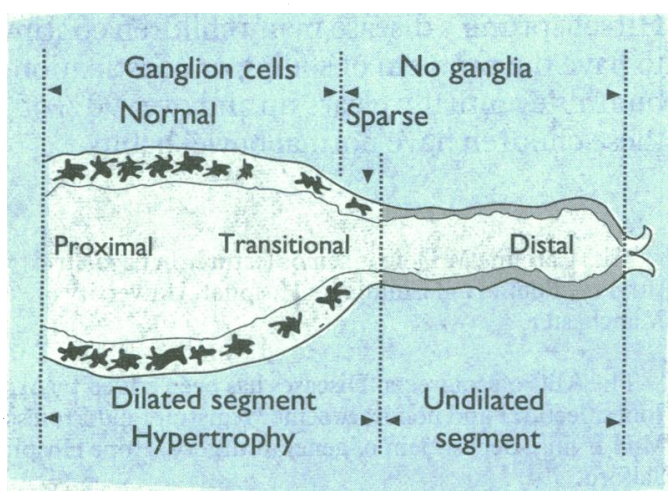

Histopathology of Hirschsprung's disease - the proximal colon is dilated and hypertrophied with ganglion cells becoming sparse at the transitional area and absent at the undilated (but not narrow) distal colon. The anus never has ganglion cells.

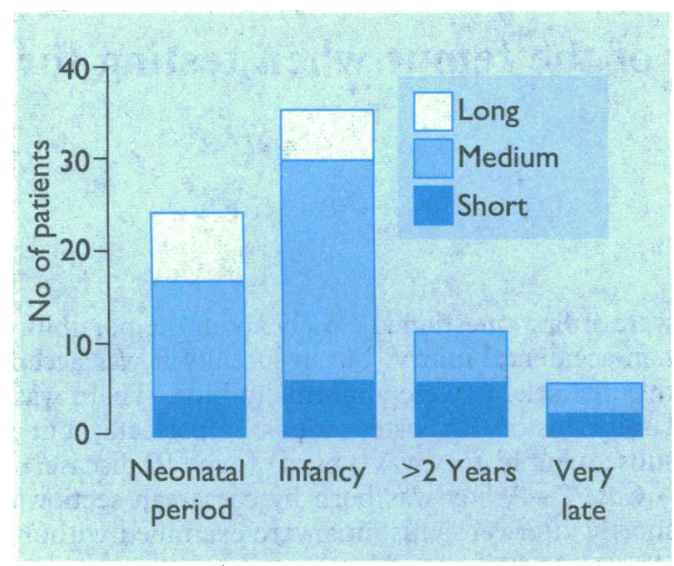

Age at presentation in relation to length of segment. (Numbers were obtained in the 1970s in London.)

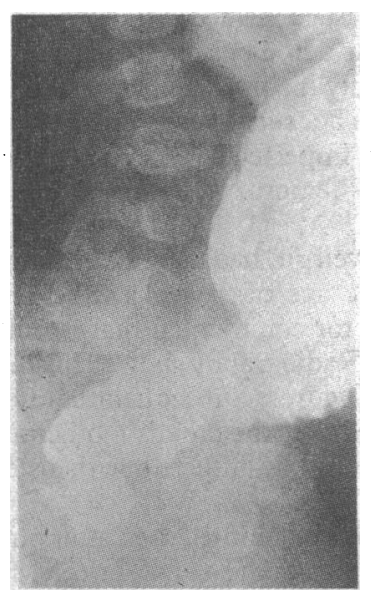

Barium enema radiograph showing cone at the transitional area with normal peristaltic dilated bowel to the aganglionic non-peristaltic normal sized bowel distally.
Rectal prolapse occurs in children under the age of 3 years. Cystic fibrosis must be excluded by a sweat test and massive intussusception can be excluded by rectal examination. By treating the constipation and reassuring the parents the prolapse will eventually cure itself. Surgery to fix the prolapse is rarely necessary.
Any child who has had constipation since birth, irrespective of age, may have Hirschsprung's disease. The disease presents either in a neonate as large bowel obstruction or later as chronic constipation. The diagnosis should be considered and excluded in any child who does not pass meconium for 48 hours or who passes a meconium plug only after rectal examination or insertion of a thermometer. The diagnosis should be considered in all constipated children, especially if constipation starts from birth or with delay in the passage of meconium. Soiling and faeces in the rectum may indicate that the disease is of short length. There is a lack of ganglion cells in Auerbach's plexus for varying lengths of bowel proximal from the anus. Total lack of ganglion cells throughout the colon or even the small bowel can occur. In children with very short or short affected segments the disease may not be diagnosed until they are teenagers. Anorectal manometry may be helpful in making the diagnosis in these children.

\section{Diagnosis}

Although it is a congenital disease, two thirds of children may be diagnosed after the age of 6 months. The diagnosis depends on radiological and histological findings. A barium enema examination is performed on the unprepared bowel. A plain abdominal radiograph 24 hours later showing retention of barium is highly suggestive. Biopsy specimens are taken from the rectum either at laparotomy or through the anus to give absolute proof - that is, the absence of ganglion cells. Histological examination of a punch biopsy specimen of mucosa and submucosa will exclude the disease if ganglion cells are present. Acetylcholinesterase activity may also be measured in these small specimens.

\section{Treatment}

The initial treatment to deflate the bowel is colostomy, which can either be in the right transverse colon if the stoma is created blind or at the junction of ganglionic and aganglionic bowel if laparotomy has been necessary to confirm the diagnosis. Although some surgeons favour deflation by rectal washouts and early neonatal definitive surgery, the usual practice is for colostomy in neonates to be followed by excision of aganglionic bowel and reanastomosis when the child weighs about $10 \mathrm{~kg}$. There are a variety of operations ("pull throughs") to reanastomose the ganglionic bowel to the normally innervated anus. The colostomy is left in place if it is in the right transverse colon and closed when the anastomosis is satisfactory or redone at surgery to divert faeces until the anastomosis is healed. 


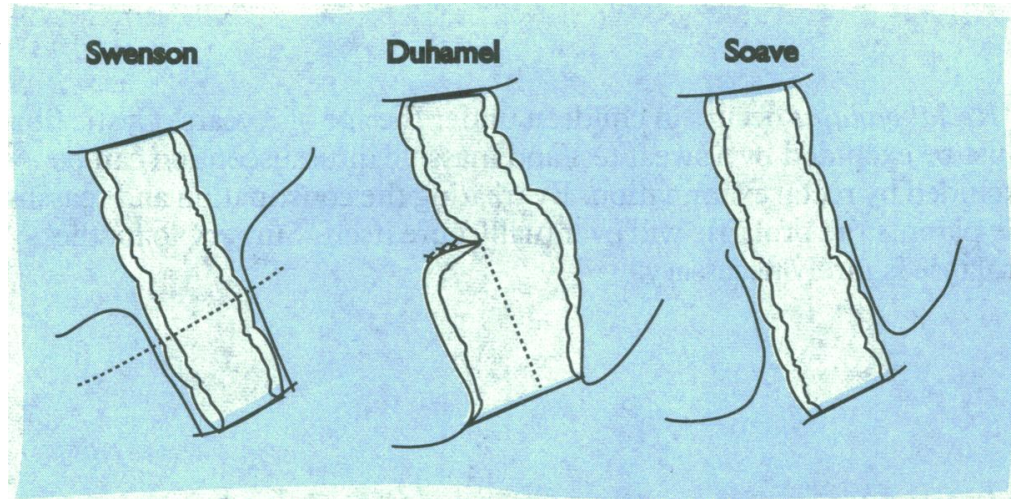

(a) Swenson operation - end to end anastomosis intussuscepting aganglionic and ganglionic bowel through anus and resection and anastomosis along dotted line. (b) Duhamel operation - anterior aganglionic and posterior ganglionic bowel. The dotted line indicates the staple closure and knife division between the two portions of bowel. (c) Soave operation-mucosa is removed, a muscular cuff of the aganglionic bowel is left and ganglionic bowel is pulled through to hang from the anus.

The photographs were prepared by the medical illustration department, North Manchester General Hospital.

\section{Complications}

The major complication and commonest cause of death in children with Hirschsprung's disease is enterocolitis which, although commoner in infancy, may occur in older children with untreated Hirschsprung's disease. It presents as a fulminating infection (often clostridial in origin) with toxaemia, abdominal distension, and diarrhoea. Despite treatment with antibiotics, intravenous fluids, or even steroids and emergency colostomy a third of these children die. Those who survive a stormy illness will have permanent mucosal damage of both ganglionic and aganglionic bowel and an increased risk of complications such as stricture and leakage after surgery. After treatment of Hirschsprung's disease many children continue to have the problem of soiling or constipation, but this eventually clears up and over $90 \%$ of these children have normal bowel habits.
Miss Caroline M Doig is senior lecturer in paediatric surgery, Booth Hall Children's Hospital, University of Manchester.

The ABC of Colorectal Diseases has been edited by $\mathrm{Mr} \mathrm{D}$ Jones, lecturer and honorary senior registrar, and Professor M H Irving, department of general surgery, Hope Hospital, Salford.

\title{
Lesson of the Week
}

\section{Osteogenesis imperfecta: fractures of the femur when testing for congenital dislocation of the hip}

\author{
Colin R Paterson, Robin J Beal, John A Dent
}

Osteogenesis imperfecta must be excluded

whenever possible before routine clinical testing

for congenital dislocation of the hip

Department of Biochemical Medicine, Ninewells Hospital and Medical School, Dundee DD1 9SY

Colin R Paterson, senior lecturer

\section{Department of}

Orthopaedic and Trauma

Surgery, Royal Infirmary, Dundee DD1 9ND

Robin J Beal, medical student John A Dent, senior lecturer

Correspondence to: Dr Paterson.
One important need in the management of osteogenesis imperfecta is the prevention of avoidable fractures. Each fracture leads to a period of immobilisation, and the consequent osteopenia may make further fractures more likely. This paper reports on a group of children whose initial fractures seem to have occurred as a result of clinical testing for congenital dislocation of the hip and seeks to identify ways in which these early fractures could be prevented.

\section{Case histories}

The patients were identified through the Brittle Bone Society, which maintains a register of patients in the United Kingdom and Ireland with known osteogenesis imperfecta. ${ }^{1}$ Patients were classified according to the Sillence scheme. ${ }^{2}$

Case 1-A girl was well until the age of 1 week, when her hips were examined by a paediatrician. She cried immediately but her mother was reassured. Subsequently, however, she continued to cry excessively when nappies were changed and she was noticed not to be moving one leg. Her mother consulted her general practitioner on three occasions, and on each of these further examinations of the hips were carried out. Eventually, at the age of 4 weeks, the left leg was noted to be swollen and after referral to the casualty department bilateral femoral fractures were identified together with tibial fractures. The parents were at first questioned closely about the possibility of non-accidental injury, but eventually it was accepted that the sclerae were abnormally blue. There was no family history of osteogenesis imperfecta, but the subsequent history was typical of type IB disease.

Case 2-A boy was born by caesarean section and shortly afterwards his hips were examined without ill effects. At 6 weeks of age he was re-examined by the health visitor at the practice surgery. Immediately he cried inconsolably and the leg swelled. After rapid referral to hospital an oblique fracture of the upper third of the femur was identified. Initially nonaccidental injury was considered because of the normal sclerae and lack of a family history. Further radiology suggested osteopenia. The subsequent history was typical of osteogenesis imperfecta type IVA; with impaired growth and 14 femoral fractures over nine years.

Case 3-A previously well girl had her hips examined by a paediatrician at the age of 9 weeks. She cried immediately and the doctor commented that he felt a "grinding" in the thighs. Radiography showed bilateral fresh femoral fractures (figure). Subsequent study of the family history showed that the child's mother and grandmother were both mildly affected with osteogenesis imperfecta type IVB.

Case 4-A girl's hips were examined soon after birth without difficulty. However, at the age of 6 weeks she was taken to another hospital for a routine check, when 